\title{
Abstract
}

\section{Homeopathic Treatment of Trauma, Abscess and Papillomatosis in Trachemys dorbigni}

\section{Bruna Scardoeli 1; Franscine Brait Narita 1; Sonia Regina Pinheiro 1; Adalberto von Ancken 1; Cidéli de Paula Coelho ${ }^{* 1,2}$}

1 - High Dilution Science (HD Science), São Paulo, Brazil

2 - Santo Amaro University, São Paulo, Brazil

*ccideli@uol.com.br - https://orcid.org/0000-0002-0492-1822

Background Mechanical trauma, bacterial and viral infections are common in Trachemys dorbigni when in captivity. Aims Report the evolution of homeopathic treatment in a patient of Trachemys dorbigni species. Methodology Homeopathic treatment was chosen, based on the principle of similitude, using Arnica montana due to its anti-inflammatory action, Avena sativa for the physical fatigue; Silicea to act in suppurative processes and Thuya occidentalis in papillomatosis. Results On $22 / 11 / 2018$, with 1 month old, 6g, it presented lethargy, hyporexia, locomotor difficulty due to edema and necrotic process in the right anterior limb, presenting bad prognosis and possible limb loss. The protocol was 2 globules of Arnica montana $6 \mathrm{CH}$, diluted in the contact water, every 8 hours, for 7 days and 2 globules of Avena sativa 6CH, every 12 hours, for 5 days. After 7 days, it was observed that the patient was active, normoretic, with weight gain and decreased edema and necrotic process of the right anterior limb, ruling out the amputation. On 29/12/2018, the patient presented a rigid abscess in the right cervical region, that adopted protocol with a single dose of 2 globules of Silicea $6 \mathrm{CH}$ and 2 globules of Arnica montana 6CH, diluted in the contact water, every 24 hours, for 4 days, until the return on 02/01/2019, with the complete regression. On 13/01/2019, it presented papillomas and plastron infection, which the proposed treatment was constituted with administration of 2 globules of Thuya occidentalis $12 \mathrm{CH}$, diluted in the contact water, every 24 hours for 3 days, with total remission of the lesions occurring in 3 days. Conclusion Mechanical trauma, bacterial and viral infections can be controlled by homeopathic medicaments without the administration of antibiotic or surgeries, being fast, less harmful and with an affordable economic value.

Keywords: homeopathy, turtle, high dilution

(C) International Journal of High Dilution Research.

Not for commercial purposes. 